\title{
Dihydrotestosterone induces p27 degradation via direct binding with SKP2 in ovarian and breast cancer
}

\author{
PENGCHENG SHI $^{1 *}$, YAN ZHANG ${ }^{3 *}$, XIAOWEN TONG $^{3}$, YU YANG $^{1,2}$ and ZHIMING SHAO ${ }^{1,2}$ \\ ${ }^{1}$ Department of Breast Surgery, Cancer Hospital, ${ }^{2}$ Institute of Biomedical Science, Fudan University, Shanghai 200032; \\ ${ }^{3}$ Department of Obstetrics and Gynecology, Tongji Hospital, Tongji University, Shanghai 200065, P.R. China
}

Received January 20, 2011; Accepted March 3, 2011

DOI: $10.3892 / \mathrm{ijmm} .2011 .677$

\begin{abstract}
The effect of androgens on the prevention or promotion of cell cycle progression in ovarian and breast cancer is controversial. This effect determines the basic rules of how to treat postmenopausal patients properly after surgery. In the present study, we investigated the effects of different androgens, namely dihydrotestosterone (DHT), testosterone and dehydroepiandrosterone (DHEA) in MCF-7 breast cancer and OVCAR-3 ovarian cancer cells. We found that DHT could down-regulate p27, but not p21, within 4 h. Further studies proved that DHT induced p27 degradation through the ubiquitin-proteasome proteolytic pathway. Inhibition of the androgen receptor and of phosphorylation did not hamper the degradation. However, proteolysis inhibitors effectively antagonized the DHT-induced p27 degradation. Knockdown of the S-phase kinase-associated protein 2 (SKP2), rather than of the Kip1 ubiquitination promoting complex 1 (KPC1), also prevented p27 down-regulation. DHT led to the direct binding of p27 to SKP2 independent of the phosphorylation status. Collectively, DHT, but not the other examined androgens regulated the degradation of p27 in MCF-7 and OVCAR-3 cells. Furthermore, SKP2 was shown to act as a DHT receptor.
\end{abstract}

\section{Introduction}

The three main androgens are dihydrotestosterone (DHT), testosterone and dehydroepiandrosterone (DHEA) (1). These androgens are steroid hormones which stimulate or control the development and maintenance of the male characteristics in vertebrates by binding to androgen receptors (ARs). Testosterone is the precursor of DHT and of all estrogens (2).

Correspondence to: Professor Zhiming Shao, Department of Breast Surgery, Cancer Hospital, Fudan University, 21st District, Dong'an Road 270, Shanghai 200032, P.R. China

E-mail: zhimingshao@yahoo.cn

*Contributed equally

Key words: dihydrotestosterone, p27, S-phase kinase-associated protein 2, direct binding, cell cycle, ovarian and breast cancer
DHT is synthesized primarily from the prostate gland, the testes, hair follicles and adrenal glands by means of reducing the 4,5 double-bond of testosterone (3). DHEA is produced in the adrenal cortex from cholesterol. It is another primary precursor of natural estrogen. Approximately 5\% of serum testosterone forms the more potent androgen, DHT. DHT has a 3 -fold greater affinity for the ARs than testosterone and a 15-fold greater affinity than adrenal androgens (4-7). Unlike testosterone and DHEA, DHT can not be converted by the enzyme aromatase to estradiol (2). Estrogen is usually present at significantly higher levels in women of reproductive age, which is also an important factor for female cancer. Aromatase inhibitors are a class of drugs used in the treatment of breast and ovarian cancer in postmenopausal women. Aromatase inhibitors block the synthesis of estrogen (8-10). Their side effect is the increase of androgen levels, especially of DHT, in the patients.

Androgens are controversial regulators of breast and ovarian cancer $(11,12)$. Estrogen has a critical role in the development and progression of breast and ovarian cancer. It has been demonstrated that androgens may prevent cancer in patients if testosterone is not converted to estrogen (8). However, other studies do not support this notion $(6,13,14)$. Moreover, the risk of breast cancer associated with elevated serum androgen levels is often explained by the structural resemblance of androgens and estrogens. However, the mechanism of androgen action in tumorigenesis remains largely unknown. There are many studies about the estrogen receptor (ER) status in cancer. The most important mechanism of tumorigenesis is the binding of estrogen to the ER, which stimulates the proliferation of mammary cells, cell division and DNA replication $(15,16)$. This is also in agreement with our previous studies $(17,18)$. However, the relationship between androgens and the ER has not been elucidated.

In the present study, we investigated the role of androgens in cancer. We found that DHT down-regulated p27, but not p21, in MCF-7 and OVCAR-3 cells within $4 \mathrm{~h}$ of treatment. This led to progression of the cell cycle. DHEA and testosterone did not induce a similar effect. Moreover, this effect was independent of the AR and the ER. Phosphorylation was not necessary for proteolysis of $\mathrm{p} 27$, while a proteasome inhibitor could prevent the degradation. DHT induced the direct binding of p27 to the S-phase kinase-associated protein 2 (SKP2). All of these results indicate that DHT may promote 
p27 interaction by binding directly to SKP2, which induces the degradation of $\mathrm{p} 27$. Further examination of the side effects of aromatase inhibitors is warranted. DHT affected the cell cycle progression independent of the AR and the ER.

\section{Materials and methods}

Antibodies and reagents. DHT, testosterone and DHEA were purchased from Sigma-Aldrich (St. Louis, MO). Anti-p27, anti-p21, anti-SKP2 and anti-Kip1 ubiquitination promoting complex 1 (anti-KPC1) antibodies were purchased from Santa Cruz Biotechnology (Santa Cruz, CA). Actinomycin D was purchased from AppliChem (Darmstadt, Germany). MG132, cycloheximide, flutamide and fulvestrant were purchased from Sigma (St. Louis, MO). Bortezomib and AZD0530 were kindly provided by Dr C. Ma from Roche (Basel, Switzerland) and from AstraZeneca (London, UK) (19), respectively. For RNAi studies, short interfering RNA (siRNA) duplexes specific for SKP2 $(\mathrm{n}=4)(1 \mu \mathrm{g}$; Dharmacon, Lafayette, CO) and KPC1 (10 nmol each; ID 00151969, 00151970 SigmaAldrich) were obtained and transfected into $\mathrm{MCF}-7$ and OVCAR-3 cells using X-tremeGENE reagent (Roche). To verify the specificity of the siRNA-mediated knockdown, scrambled siRNA was transfected in parallel. All chemical reagents were diluted in DMSO, ethanol or water.

Cell culture. MCF-7 and OVCAR-3 cells were maintained in Dulbecco's modified Eagle's medium (Thermo Hyclone, Candler, NC) containing $10 \%$ fetal bovine serum (FBS). The cells were starved of androgen for $8 \mathrm{~h}$ and incubated in DMEM medium with 5\% charcoal-stripped FBS (Biological Industries, Kibbutz Beit Haemek, Israel) and $1 \mathrm{nmol}_{2}$ (estrogen, Sigma-Aldrich) in $37^{\circ} \mathrm{C}$ and $5 \% \mathrm{CO}_{2}$. Cells were cultured until $70 \%$ confluency before the treatment.

Western blot analysis. Cell lysates were prepared in RIPA buffer with protease inhibitors (cOmplete mini protease inhibitor, Roche) and phosphatase inhibitor cocktail (SigmaAldrich). The lysates were then clarified by centrifugation at $12,000 \mathrm{x}$ g for $10 \mathrm{~min}$ at $4^{\circ} \mathrm{C}$. Protein concentration was measured by the BCA assay (Thermo Scientific). Equivalent amounts of protein were subjected to SDS-PAGE analysis. Immunoblotting was performed using standard methods. Quantification of the protein was conducted by the Image Analyzer LAS-3000 (FujiFilm Life Science, Tokyo, Japan). The image was analyzed by densitometry. Each sample was normalized to GAPDH.

Flow cytometry analysis of the cell cycle. Cells were cultured in DMEM with $10 \mathrm{nmol} / \mathrm{l}$ DHT for $6 \mathrm{~h}$. Bromodeoxyuridine (BrdU) $(10 \mu \mathrm{l}$ of a $9 \mathrm{mg} / \mathrm{ml})$ solution was added to $5 \mathrm{ml}$ of medium for $30 \mathrm{~min}$ before harvesting the cells. The protocol was based on a previous study (20). The cells were collected by trypsinization, centrifuged at $1,600 \mathrm{x} \mathrm{g}$ for $6 \mathrm{~min}$, fixed with $70 \%$ ethanol overnight and analyzed for cell cycle distribution by propidium iodide/BrdU bivariate flow cytometry.

Real-time analysis. Total RNA was extracted using the TRIzol reagent (Invitrogen, Carlsbad, CA). First-strand cDNAs were synthesized using SuperScript III Reverse Transcriptase
(Takara, Dalian, China) with oligo(dT) primers. The primer sequences were as follows: human p27 (forward primer, 5'-AGATGTCAAACGTGCGAGTG-3' and reverse primer, 5'-TCTCTGCAGTGCTTCTCCAA-3'); human GAPDH (forward primer, 5'-TGCCAAATATGATGACATCAAGAA-3' and reverse primer, 5'-GGAGTGGGTGTCGCTGTTG-3'). The iCycler and iQ real-time PCR (Bio-Rad, Hercules, CA) and SYBR-Green PCR Master mix (Toyobo, Osaka, Japan) were used for amplification and detection. The qPCR conditions were as follows: $95^{\circ} \mathrm{C}$ for $5 \mathrm{~min}$; and $95^{\circ} \mathrm{C}$ for $30 \mathrm{sec}$, $60^{\circ} \mathrm{C}$ for $30 \mathrm{sec}$ and $72^{\circ} \mathrm{C}$ for $30 \mathrm{sec}$ for 39 additional cycles. The qPCR results were presented as threshold cycle $(\mathrm{Ct})$ value and the relative mRNA level was analyzed by normalizing to GAPDH.

Pull-down assay. For the pull-down assay, the p27 gene (Origene, CA) was constructed into pGEX-6p-1 and p27-GST fusion proteins were purified with GST-sepharose beads (GE Healthcare, NY). The purified protein was washed with TEE buffer (50 mM Tris pH 7.9, 1 mM EDTA, 1 mM EGTA). For the pull-down assays, $1 \mu \mathrm{g}$ of p27-GST and $4 \mu \mathrm{g}$ of SKP2 (Genscript, Nanjin, China) were added to the buffer $(0.15 \mathrm{M}$ $\mathrm{NaCl}, 0.5 \%$ Nonidet $\mathrm{P}-40,0.1 \mathrm{M}$ Tris- $\mathrm{HCl} \mathrm{pH}$ 7.5, containing $10 \mathrm{mM} \beta$-glycerophosphate, $1 \mathrm{mM}$ phenylmethylsulphonyl fluoride, $1 \mathrm{mM}$ dithiothreitol, $1 \mathrm{mM} \mathrm{NaF}, 10 \mathrm{mM}$ MG132 and $2 \mathrm{mM}$ pepstatin A) with DHT treatments as indicated. The reactions were incubated with additional glutathione beads for $30 \mathrm{~min}$ and washed 3 times in the buffer with the corresponding DHT concentration. Glutathione beads were recovered. Pulldown mixtures were separated on SDS-polyacrylamide gel electrophoresis (PAGE) and the bound SKP2 was detected by Western blot analysis. The immunodetection of SKP2 was performed with a 1:200 dilution of the polyclonal anti-SKP2 antibody (Santa Cruz), followed by a 1:4,000 dilution of the goat anti-rabbit IgG-peroxidase conjugate (Kangchen, Shanghai, China), and chemiluminescent detection with the West Femto ECL plus reagents (Pierce, Clearwater, ID).

\section{Results}

DHT reduces $p 27$. To examine the relationship between androgens and the cell cycle progression in female malignant tumors, we screened the effects of various androgens in MCF-7 and OVCAR-3 cells. Both cell lines were cultured in DMEM with 5\% charcoal-stripped serum for $8 \mathrm{~h}$ before use. DHT reduced p27 protein levels within $4 \mathrm{~h}$, but the other androgens did not show similar results (Fig. 1A, C and D). DHT up-regulated the expression of p27 after $24 \mathrm{~h}$. This up-regulation could also be observed with DHEA and testosterone. However, DHT did not down-regulate p21 (Fig. 1A), similarly to DHEA and testosterone. The effects of androgens on the cell cycle progression of MCF-7 and OVCAR-3 cells was examined by FACS analysis. After $5 \mathrm{~h}$ of DHT treatment, MCF-7 cells in the S phase increased from 20.1 to $29.2 \%$ (Fig. 1B). The respective increase in OVCAR-3 cells was from 11.2 to $18.1 \%$. Accordingly, the percentage of cells in the G1 phase decreased. DHT showed a time-dependent regulation of the cell cycle progression. Increases in the concentration of DHT strengthened the p27 down-regulation, but the doseeffect relationship was not linear (Fig. 1E). After 24 h, DHT 
A
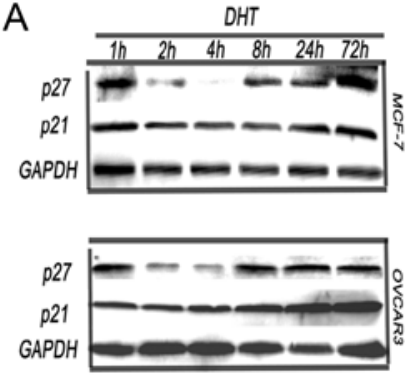

C

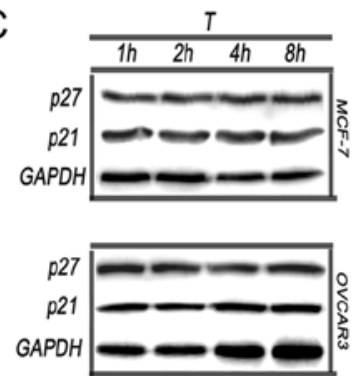

B

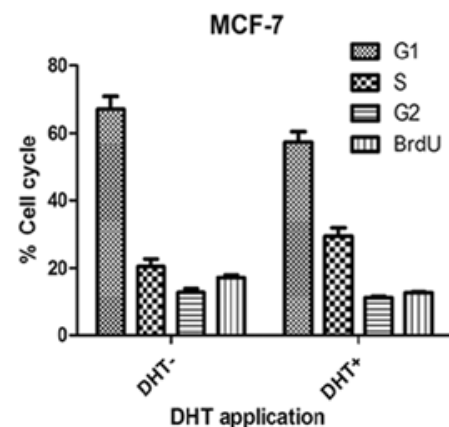

D

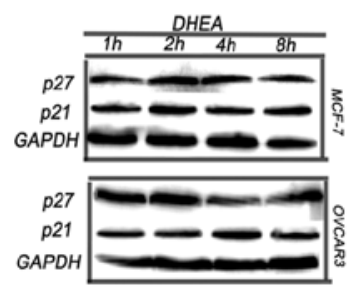

OVCAR3

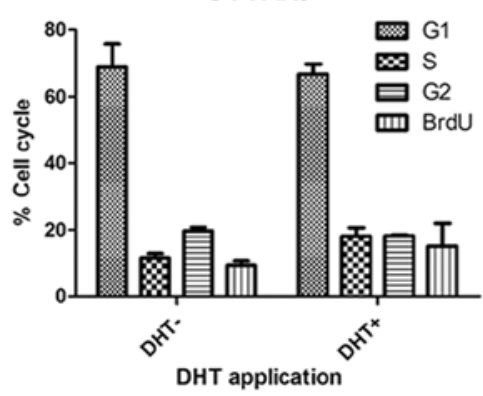

$E$

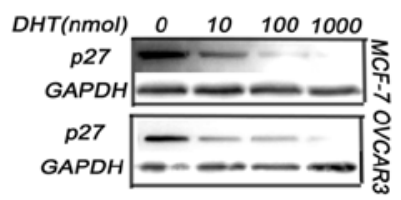

Figure 1. Effect of different androgens on protein levels of p21 and p27 and on the cell cycle progression. Western blot analysis demonstrated that DHT (10 nmol) down-regulated p27, but not p21, within $4 \mathrm{~h}$ of treatment in the MCF-7 and OVCAR-3 cell lines. The effect of DHT (10 nmol) on the cell cycle progression was detected by propidium iodide/BrdU-bivariate flow cytometric analyses. Cells were exposed to DHT for $6 \mathrm{~h}$. BrdU was added for the last 30 min to label S-phase cells active in DNA replication. Values are the mean \pm standard error of the mean $(\mathrm{n}=4)$. (C and D) Western blot analysis demonstrated that testosterone (T, $10 \mathrm{nmol})$ and DHEA (10 nmol) did not down-regulate p27 and p21 within $4 \mathrm{~h}$ in the MCF-7 and OVCAR-3 cell lines. (E) Down-regulation of p27 by DHT ( $10 \mathrm{nmol})$ was concentration-dependent, but the relationship was not linear.

up-regulated p27 in agreement with a previous study (21). DHT had a paradoxical effect on the cell cycle progression of female cancer. Moreover, DHEA and testosterone did not inhibit or promote this effect. Collectively, these results indicate that DHT has a unique function in the initiation of cell division, since p27 is a crucial checkpoint of G0/G1. This function is totally different from that of the other androgens examined.

DHT down-regulates p 27 by controlling its degradation. In this context, we attempted to ascertain whether p27 was controlled by DHT at the mRNA, protein or degradation level. Actinomycin D is primarily used as an investigative tool in cell biology to inhibit transcription because it can prevent elongation of the mRNA (22). Compared to the control group, DHT decreased p27 even in the presence of $1.0 \mu \mathrm{g} / \mathrm{ml}$ actinomycin D (Fig. 2A). However, DHT did not down-regulate p27 at the mRNA level within $4 \mathrm{~h}$ (Fig. 2E). We next used cycloheximide to determine the half-life of p27 (Fig. 2F). We found that p27 was not detectable after just $4 \mathrm{~h}$ of treatment. p27 is mainly degraded through the proteolysis and ubiquitination pathways. MG132 is a specific, potent and cell-permeable inhibitor used in many studies. Bortezomib is the first therapeutic inhibitor to be used in patients with refractory or rapidly advancing disease. Both of these inhibitors could eliminate the DHT-induced down-regulation of p27 (Fig. 2C and D). On the other hand, cycloheximide accelerated the DHT-induced p27 down-regulation. It therefore appears that DHT reduces p27 within $4 \mathrm{~h}$ via a proteolysis pathway. Generally, phosphorylation of several peptides of p27 is essential for degradation via the proteasome. Serine 10 (Ser10) phosphorylation often leads to stability of the cell cycle. This notion is not in agreement with our present results (Fig. 1A). We therefore, used AZD0530 to examine the phosphorylation status. In the present study we found that inhibition of phosphorylation did not prevent p27 degradation (Fig. 2B).

DHT decreases 27 through SKP2 without affecting its phosphorylation. Previous research has demonstrated that testosterone and DHT may strengthen the expression of p27 or attenuate its degradation via the proteasome. In the present study, we used the AR inhibitor, flutamide, in order to examine whether the AR is involved in the p27 decrease observed within $4 \mathrm{~h}$ (Fig. 3A). Our results indicate that flutamide does not influence the degradation of p27. Different concentrations of flutamide hardly affected the degradation of p27. We also examined the effect of the ER inhibitor, fulvestrant, on p27 degradation (Fig. 3B). We obtained similar results to those with the AR inhibitor. There are two main E3 ligases, SKP2 and KPC, that have been reported to be responsible for $\mathrm{p} 27$ degradation (23). Because of the similarities between KPC1 and KPC2, SKP2 and KPC1 were chosen to be examined in our study. SKP2 siRNA, but not KPC1 siRNA, affected the degradation of p27 (Fig. 3C and D). These results indicate that DHT down-regulates p27 through a SKP2-controlled pathway. Therefore, the DHT-induced decrease of p27 within $4 \mathrm{~h}$ occurs via a SKP2-controlled proteolysis pathway and is independent of phosphorylation.

DHT leads to direct binding of p27 to SKP2. The Auxin-IAASCR-TIR1 interaction does not require stable modification of the protein $(24,25)$. The structural similarity of the molecules 
A
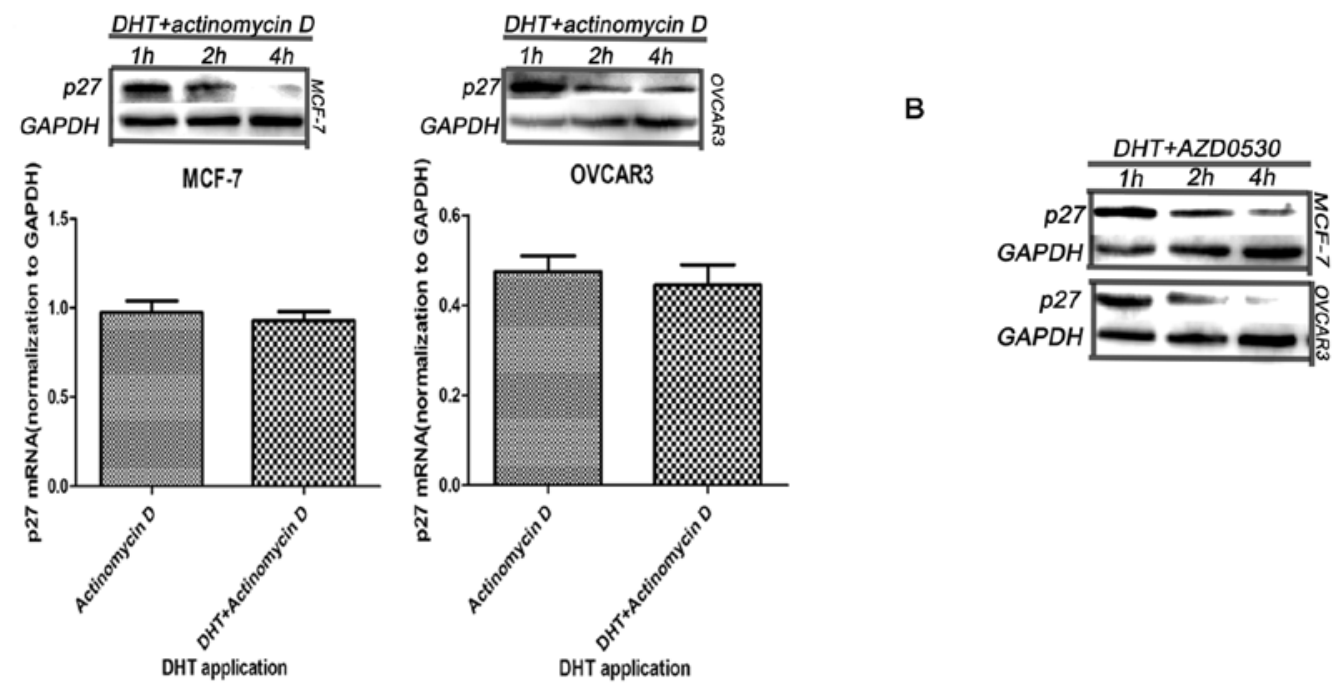

C

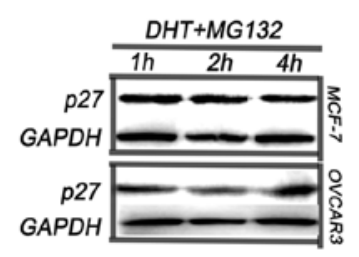

D
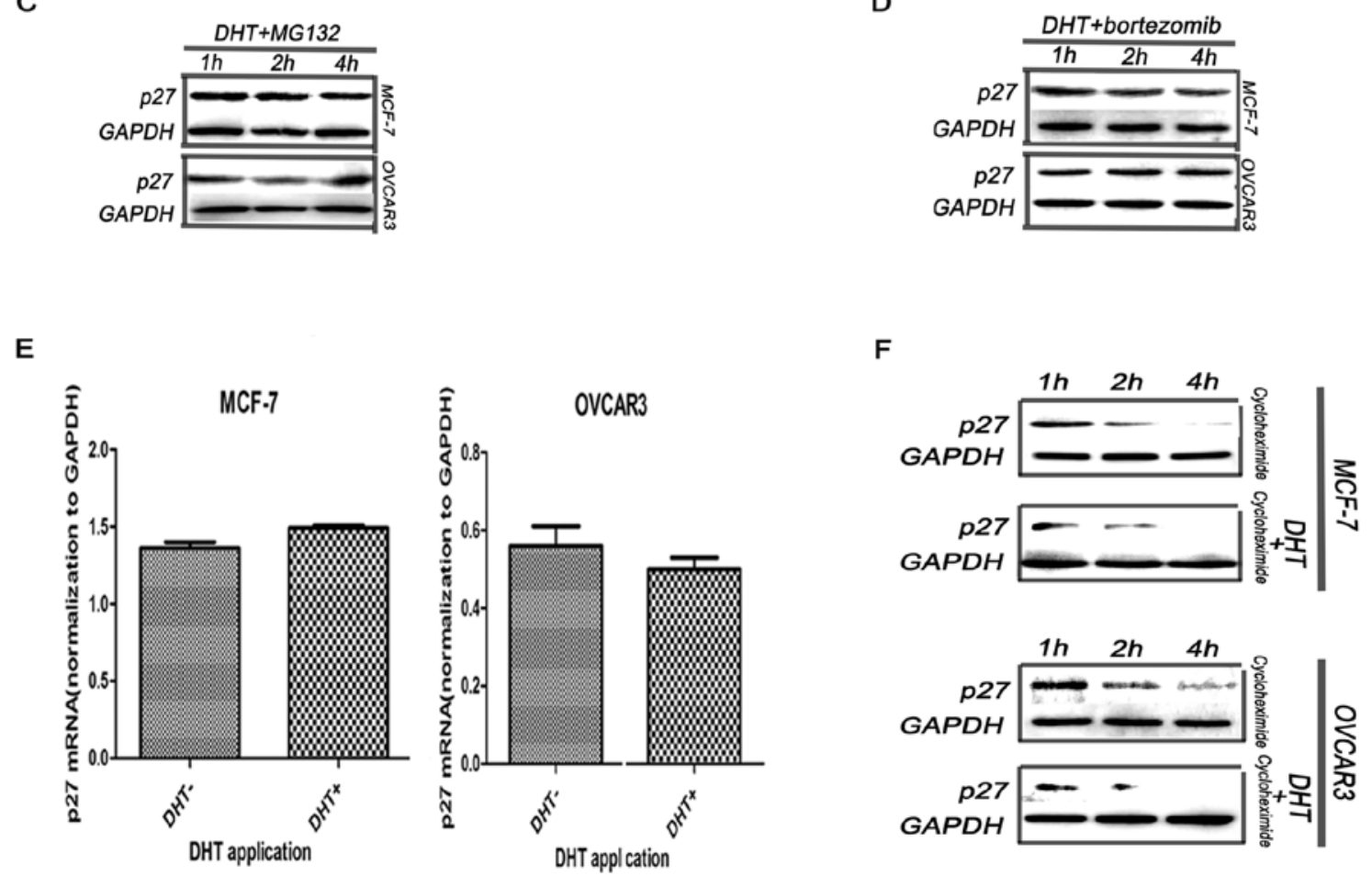

Figure 2. Effect of DHT in combination with different inhibitors on p27 expression in MCF-7 and OVCAR-3 cells. (A and B) Western blot analysis demonstrated that actinomycin D (1.0 $\mu \mathrm{g}$, a DNA transcription suppressor) and AZD0530 (1 $\mu \mathrm{mol}$, a phosphorylation inhibitor) do not influence the down-regulation of p27 by DHT (10 nmol) in either cell line within $4 \mathrm{~h}$. The lower panel indicates the normalization to GAPDH. (C and D) Western blot analysis showed that the proteasome inhibitors (MG132, $10 \mu \mathrm{mol}$; bortezomib, $1 \mathrm{mg}$ ) prevented the degradation of p27 by DHT (10 nmol) in MCF-7 and OVCAR-3 cell lines within $4 \mathrm{~h}$. (E) DHT (10 nmol) did not have an effect on p27 mRNA expression within $4 \mathrm{~h}$ as determined by qPCR. (F) Cycloheximide (2 $\mu \mathrm{g} / \mathrm{ml}) \mathrm{did}$ not inhibit the DHT (10 nmol)-induced p27 degradation, but rather accelerated this process.

investigated in the present study with auxin and inositol hexakisphosphate together with the above-mentioned results indicating that p27 down-regulation by DHT is independent of phosphorylation and the AR or ER, prompted us to hypothesize that DHT might regulate the interaction between p27 and SKP2 to form a directly binding complex. A pull-down assay was performed with cell lysates or expressed protein at different temperatures and different DHT concentrations (Fig. 4A and B). The direct binding induced by DHT was independent of the temperature and dependent on the DHT concentration. The reaction was carried out at $4,10,20$ or $37^{\circ} \mathrm{C}$ (Fig. 4A). As the concentration of DHT increased, the binding of p27 to SKP2 increased (Fig. 4B). Testosterone and DHEA did not exert this effect (data not shown). In a subsequent experiment, cell lysates extracted from MCF-7 and OVCAR-3 cells were subjected to immunoprecipitation with exogenous GST-p27 (Fig. 4C). An increase of SKP2 precipitation with DHT addition was observed. These results support the notion that DHT down-regulates p27 via promoting direct binding between $\mathrm{p} 27$ and SKP2. 


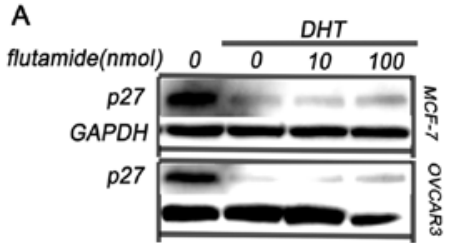

C

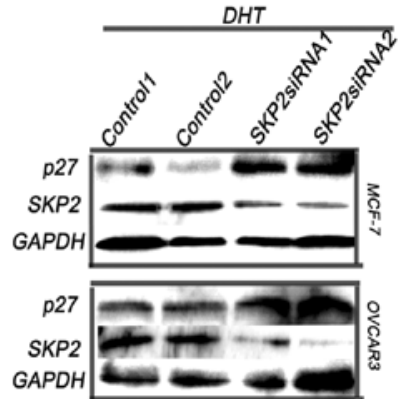

B

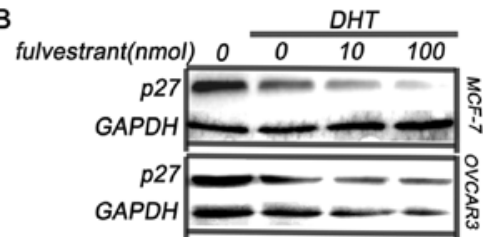

D

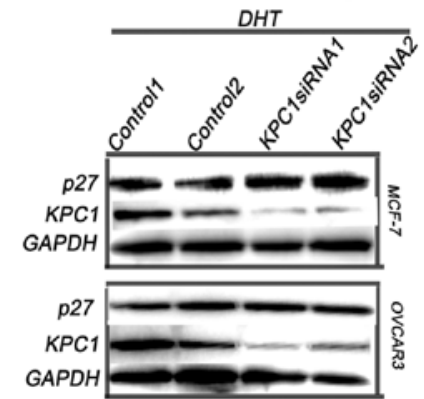

Figure 3. Effect of DHT treatment in combination with various steroid receptor inhibitors or with siRNAs for two different E3 ligases (SKP2 or KPC1) on p27 expression in MCF-7 and OVCAR-3 cells. (A and B) Western blot analysis showed that flutamide (an AR inhibitor) or fulvestrant (an ER inhibitor) at various concentrations did not influence the down-regulation of p27 by DHT (10 nmol) in MCF-7 or OVCAR-3 cell lines within 4 h. (C) Western blot analysis showed that SKP2 siRNA transfection successfully reduced SKP2 levels and prevented the degradation of p27 by DHT (10 nmol) in MCF-7 and OVCAR-3 cell lines within $4 \mathrm{~h}$. (D) Western blot analysis showed that KPC1 siRNA transfection successfully reduced KPC1, but did not prevent the degradation of p27 by DHT $(10 \mathrm{nmol})$ in MCF-7 and OVCAR-3 cell lines within $4 \mathrm{~h}$.

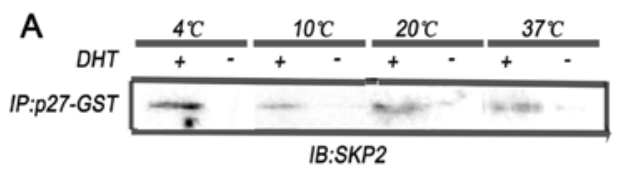

B

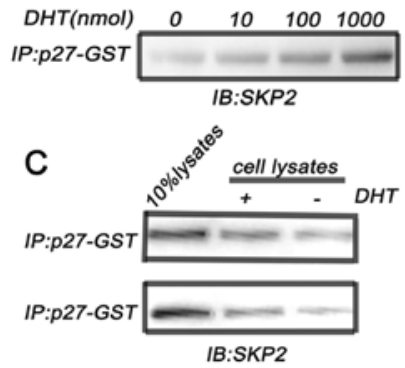

Figure 4. Pull-down assay performed for exogenous or endogenous p27 and SKP2. (A) Pull-down reactions were carried out at the indicated temperatures for $30 \mathrm{~min}$ in the presence or absence of DHT. (B) Pull-down reactions were carried out for the indicated times with different DHT concentration at $4{ }^{\circ} \mathrm{C}$. (C) Cell lysates from MCF-7 or OVCAR-3 were immunoprecipitated by exogenous p27 (p27-GST) and immunoblotted by SKP2, with or without DHT $(10 \mathrm{nmol})$.

\section{Discussion}

Haploinsufficiency occurs when a diploid organism has only a single functional copy of a gene and does not produce enough of the product (typically a protein) thus bringing about an abnormal or diseased state (26). In a murine model, p27 is a haploinsufficient gene for tumor suppression (27). Many studies also support that p27 has a pivotal role in cancer progression (10). Down-regulation of p27 promotes the development of different types of cancer. DHT is an androgen which affects both men and women. It might be life-threatening for human beings. Nevertheless, there are many investigations about the role of the AR in cancer. Many studies demonstrate that androgens and the AR protect patients from cancer. Androgens and the AR have been shown to up-regulate $\mathrm{p} 27$ when MCF-7 cells are treated with an androgen for $24 \mathrm{~h}$ (21). Estrogen and the ER have been reported to induce SKP2 expression $(28,29)$. There is no evidence indicating that DHT decreases SKP2 via binding to the ER. Therefore, the decrease of p27 by DHT in our present study may have an effect not only on female cancer but also on lung or clone cancer $(30,31)$.

Estrogen has gained enough attention in breast and ovarian cancer. There are many studies on the role of androgens in prostate cancer. Compared to estrogen, relatively few investigators are interested in the relationship between DHT and female malignant tumors. However, androgens are a rather important factor for the psychological function even in women. Treatment with androgens alone has led to androgendependent mammary carcinogenesis in transgenic rats with high expression of erbb2 (32). Androgens, including DHT or testosterone, are indispensable for mammary carcinogenesis in male or female rats with an amplified erbb2 gene. However, the carcinogenesis is not associated with the somatic mutational activation of erbb2 in the erbb2 transgenic rat. DHT, but not testosterone, has been shown to increase cancer progression in AR-negative animal models.

Phosphorylation of p 27 is considered a prerequisite of ubiquitination. Ser(10) and $\operatorname{Thr}(187)$ have been shown to be important for p27(Kip1) function $(33,34)$. Ubiquitination commonly depends on modification of a peptide. It is atypical that the auxin protein binds to Tirl directly with the help of auxin and inositol hexakisphosphate. This finding solved the puzzle about the mechanism of plant growth $(24,25)$. In human beings, p27 and SKP2 exist in different cellular compartments (35). This ameliorates the effect of p27 degradation by DHT. Besides, the concentration of DHT in the human body is not as 
high as that of testosterone. The degradation of p27 by DHT is more moderate than the down-regulation of auxin protein by auxin. The F-box protein is a huge family. E1, E2 and E3 are different proteins involved in different protein degradation pathways. Sexual hormones or the intake of similar factors from the external environment may function as DHT in the ubiquitination pathway.

In this study, we demonstrated that DHT up-regulates p27 independent of phosphorylation. Nevertheless, it should be kept in mind that p27 is just one of the checkpoint proteins. The current study demonstrated that $\mathrm{p} 21$ was not regulated by the same mechanism as p27 (Fig. 1A). Other proteins involved in the cell cycle are also important for proliferation. Moreover, DHT was shown to trigger p27 degradation in MCF-7 and OVCAR-3 cells.

In summary, p27 could be down-regulated by DHT via direct binding and was independent of phosphorylation. This effect is universal at least in female cancer. For breast cancer or ovarian cancer patients, the benefits and risks from aromatase inhibitors should be balanced, especially for the AR-negative patients.

\section{Acknowledgements}

This study was funded by the Chinese National Program for Fundamental Research and Development (973 Program) and the Chinese National Natural Science Foundation to Professor Zhiming Shao.

\section{References}

1. Vermeulen A: Plasma levels and secretion rate of steroids with anabolic activity in man. Environ Qual Saf Suppl 5: 171-180, 1976.

2. Burger HG: Androgen production in women. Fertil Steril 77 (Suppl. 4): S3-S5, 2002.

3. Luu-The V and Labrie F: The intracrine sex steroid biosynthesis pathways. Prog Brain Res 181: 177-192, 2010.

4. Jegou B, Dacheux JL, Terqui M, et al: Studies of the androgen binding protein in the rete testis fluid of the ram and its relation to sexual season. Mol Cell Endocrinol 9: 335-346, 1978.

5. Sawaya ME, Honig LS and Hsia SL: Increased androgen binding capacity in sebaceous glands in scalp of male-pattern baldness. J Invest Dermatol 92: 91-95, 1989.

6. Hida N, Poulin R, Veilleux R and Labrie F: Differential androgen sensitivity is associated with clonal heterogeneity in steroid metabolism, ornithine decarboxylase regulation and IL-1alpha action in mouse mammary tumor cells. J Steroid Biochem Mol Biol 71: 71-81, 1999.

7. Bellemare V, Faucher F, Breton R and Luu-The V: Characterization of 17alpha-hydroxysteroid dehydrogenase activity (17alpha-HSD) and its involvement in the biosynthesis of epitestosterone. BMC Biochem 6: 12, 2005.

8. Stoner E: The clinical development of a 5 alpha-reductase inhibitor, finasteride. J Steroid Biochem Mol Biol 37: 375-378, 1990.

9. Mani A and Gelmann EP: The ubiquitin-proteasome pathway and its role in cancer. J Clin Oncol 23: 4776-4789, 2005.

10. Chu IM, Hengst L and Slingerland JM: The Cdk inhibitor p27 in human cancer: prognostic potential and relevance to anticancer therapy. Nat Rev Cancer 8: 253-267, 2008.

11. Suzuki T, Miki Y, Takagi K, et al: Androgens in human breast carcinoma. Med Mol Morphol 43: 75-81, 2010.

12. Nam EJ and Kim YT: Alteration of cell-cycle regulation in epithelial ovarian cancer. Int J Gynecol Cancer 18: 1169-1182, 2008.
13. Lin HY, Sun M, Lin C, et al: Androgen-induced human breast cancer cell proliferation is mediated by discrete mechanisms in estrogen receptor-alpha-positive and -negative breast cancer cells. J Steroid Biochem Mol Biol 113: 182-188, 2009.

14. Krysiak R, Frysz-Naglak D and Okopien B: Current views on the role of dehydroepiandrosterone in physiology, pathology and therapy. Pol Merkur Lekarski 24: 66-71, 2008 (In Polish).

15. Santen R, Cavalieri E, Rogan E, et al: Estrogen mediation of breast tumor formation involves estrogen receptor-dependent, as well as independent, genotoxic effects. Ann NY Acad Sci 1155: 132-140, 2009.

16. Miyoshi Y, Murase K, Saito M and Oh K: Prediction of hormone sensitivity for breast cancers. Breast Cancer 17: 86-91, 2010.

17. Fan L, Zheng Y, Yu KD, et al: Breast cancer in a transitional society over 18 years: trends and present status in Shanghai, China. Breast Cancer Res Treat 117: 409-416, 2009.

18. Yin W, Di G, Zhou L, et al: Time-varying pattern of recurrence risk for Chinese breast cancer patients. Breast Cancer Res Treat 114: 527-535, 2009.

19. Ma C, Niu X, Luo J, et al: Combined effects of lapatinib and bortezomib in human epidermal receptor 2 (HER2)overexpressing breast cancer cells and activity of bortezomib against lapatinib-resistant breast cancer cells. Cancer Sci 101: 2220-2226, 2010

20. Malewicz B, Wang Z, Jiang C, et al: Enhancement of mammary carcinogenesis in two rodent models by silymarin dietary supplements. Carcinogenesis 27: 1739-1747, 2006.

21. Greeve MA, Allan RK, Harvey JM and Bentel JM: Inhibition of MCF-7 breast cancer cell proliferation by 5alpha-dihydrotestosterone; a role for p21(Cip1/Waf1). J Mol Endocrinol 32: 793-810, 2004.

22. Sobell HM: Actinomycin and DNA transcription. Proc Natl Acad Sci USA 82: 5328-5331, 1985.

23. Kamura T, Hara T, Matsumoto M, et al: Cytoplasmic ubiquitin ligase KPC regulates proteolysis of p27(Kip1) at G1 phase. Nat Cell Biol 6: 1229-1235, 2004.

24. Kepinski S and Leyser O: The Arabidopsis F-box protein TIR1 is an auxin receptor. Nature 435: 446-451, 2005.

25. Dharmasiri N, Dharmasiri S and Estelle M: The F-box protein TIR1 is an auxin receptor. Nature 435: 441-445, 2005.

26. Berger AH and Pandolfi PP: Haplo-insufficiency: a driving force in cancer. J Pathol 223: 138-147, 2011.

27. Fero ML, Randel E, Gurley KE, et al: The murine gene p27Kip1 is haplo-insufficient for tumour suppression. Nature 396: 177-180, 1998.

28. Signoretti S, Di Marcotullio L, Richardson A, et al: Oncogenic role of the ubiquitin ligase subunit Skp2 in human breast cancer. J Clin Invest 110: 633-641, 2002.

29. Strom A and Hartman J: Estrogen receptor beta inhibits 17betaestradiol-stimulated proliferation of the breast cancer cell line T47D. Proc Natl Acad Sci USA 101: 1566-1571, 2004

30. Berta L, Fronticelli Baldelli C, Fazzari A, et al: Sex steroid receptors, secondary bile acids and colorectal cancer. A possible mechanism of interaction. Panminerva Med 45: 261-266, 2003.

31. Provost PR, Blomquist CH, Godin C, et al: Androgen formation and metabolism in the pulmonary epithelial cell line A549: expression of 17 beta-hydroxysteroid dehydrogenase type 5 and 3alpha-hydroxysteroid dehydrogenase type 3. Endocrinology 141: 2786-2794, 2000.

32. Watson PA, Kim K, Chen KS and Gould MN: Androgendependent mammary carcinogenesis in rats transgenic for the Neu proto-oncogene. Cancer Cell 2: 67-79, 2002.

33. Zhou W, Yang Q, Low CB, et al: Pin1 catalyzes conformational changes of Thr-187 in p27Kip1 and mediates its stability through a polyubiquitination process. J Biol Chem 284: 23980-23988, 2009.

34. Kajihara R, Fukushige S, Shioda N, et al: CaMKII phosphorylates serine 10 of p27 and confers apoptosis resistance to HeLa cells. Biochem Biophys Res Commun 401: 350-355, 2010.

35. Borriello A, Cucciolla V, Criscuolo M, et al: Retinoic acid induces p27Kip1 nuclear accumulation by modulating its phosphorylation. Cancer Res 66: 4240-4248, 2006. 